\title{
Estudios CTS sobre las tendencias de las Tecnologías de la Información y las Comunicaciones aplicadas al Turismo
}

DOI: https://doi.org/10.33262/ap.v3i2.1.54

\begin{abstract}
(c) (i) (ㅇ) BY NC SA

CTS studies on trends in information and communication technologies applied to tourism
\end{abstract}

Edgar Nuñez Torres. ${ }^{1}$, Luis Pérez Rives. ${ }^{2}$, Elio Tomás Montes de Oca Companioni. ${ }^{3}$ \& Luis Efraín Velastegui López. ${ }^{4}$

\begin{abstract}
.
The New Information and Communication Technologies (ICT) have modified the current scenaries for the commercialization of tourism products on a global scale, becoming an essential route in the short, medium and long-term development of emerging tourist regions, as well as destinations highly demanded. These technologies are a catalyst for tourist flows, as they enhance the cultural values and attributes of any offer or product in the international tourism market. This work delves into holistic and specific aspects of the advancement of ICTs, as well as the importance and impact for tourism from conceptual and practical perspectives on their application. Similarly, research converges in universes of science, technology and information society, factors that help to understand from triangulation method, the contribution of ICT to the improvement and
\end{abstract}

\footnotetext{
${ }^{1}$ Facultad de Turismo, Universidad de La Habana. Cuba, enunez8609@gmail.com

2 Sucursal Marina Marlin Cayo Largo del Sur. Cuba. lperez910329@gmail.com

${ }^{3}$ Hotel Paradisus Cayo Santa María. Cuba.. eliomontes64@gmail.com

${ }^{4}$ Ciencia Digital Editorial, Ecuador, luisefrainvelastegui@cienciadigital.org
} 
evolution of tourism through the use of eTourism "or" online tourism " in the different systems and processes of the tourism industry.

Keywords: CTS, TIC, tourism:

\section{Resumen.}

Las nuevas Tecnologías de la Información y las Comunicaciones (TIC) han modificado los escenarios actuales de comercialización de productos turísticos a escala global, constituyéndose en una vía esencial en el desarrollo a corto, mediano y largo plazo de regiones turísticas emergentes, así como en destinos altamente demandados. Estas tecnologías son elemento catalizador de los flujos turísticos, pues realizan los valores culturales y atributos de cualquier oferta o producto en el mercado turístico internacional. El presente trabajo profundiza aspectos holísticos y específicos sobre el avance de las TIC, así como la importancia e impacto para el turismo desde perspectivas conceptuales y prácticas sobre la aplicación de las mismas. De igual forma la investigación converge en universos de la ciencia, tecnología y sociedad de la información, factores que ayudan a comprender desde la triangulación el aporte de las TIC al perfeccionamiento y evolución del turismo mediante el aprovechamiento del "eTurismo" o "turismo on line" en los diferentes sistemas y procesos de la industria turística.

Palabras claves: CTS, TIC, Turismo

\section{Introducción.}

Con la evolución y avance de la tecnología en los últimos años, el sector del turismo se ha visto bastante beneficiado ya que en años anteriores el flujo de información era muy escaso. Con la facilidad que tiene cualquier persona de acceder a la información hoy en día, el mercado turístico cuenta con diversas herramientas para promocionar sus productos, cosa que era un poco difícil en años anteriores ya que la tecnología estaba limitada.

El impacto de las Tecnologías de la Información y las Comunicaciones (TIC) en el turismo ha ocupado un lugar muy importante y necesario entre los distintos actores. Esta importancia se debe a la naturaleza de los productos y servicios turísticos, la cual está dada por las condiciones de producción y reproducción del modelo de gestión de la actividad.

Las nuevas Tecnologías de la Información y de la Comunicación (TIC) han contribuido decisivamente al crecimiento masivo del turismo y al aumento de valor de la oferta y de la demanda. Como ocurre con cualquier producto, en el caso del turismo la información forma parte del propio interés turístico, como uno más de sus factores de producción. Pero el uso de las TIC no es propiamente de actualidad, con anterioridad, las empresas 
turísticas ya venían haciendo uso de las nuevas tecnologías desde mediados del siglo pasado.

En un primer momento a través de las CRS (Computer Reservation Systems), que permitían a las compañías aéreas la gestión automática de reservas. Posteriormente, con la proliferación de diversos CRS -incompatibles entre sí-, en la década de los ochenta se crean los sistemas GDS (Global Distribution Systems), como sistema global de distribución que van a comprender a las diferentes compañías aéreas.

GDS es una red informática grande y, al mismo tiempo, una herramienta de reserva que transfiere el inventario y las tarifas de los hoteles que permite realizar reservas, es una red avanzada que se especializa en vender y reservar productos de viaje, como habitaciones y paquetes de servicios. Cuando un operador hotelero se conecta a un GDS, el operador puede acceder a todos los servicios dentro de la red GDS. (Fernández Álvarez, 2015, p. 2)

De esta forma, los clientes pueden reservar fácilmente habitaciones y paquetes. Hay muchos tipos de GDS, pero es fundamental que los hoteles trabajen con un GDS en la industria hotelera. Un GDS entiende las necesidades y objetivos únicos de la industria hotelera y de los operadores que gestionan propiedades de todos los tamaños. Hoy en día estos sistemas suministran información de forma rápida y precisa sobre las diferentes asociaciones de proveedores de los servicios turísticos, compuestos por compañías aéreas y cadenas hoteleras.

El turismo electrónico ("eTurismo" o "turismo on line") radica en el acceso virtual de todos los procesos: alojamiento, transporte, ocio, viajes, restauración, intermediación y servicios complementarios. Este concepto incluye todas las funciones del negocio turístico: el marketing, las finanzas, la contabilidad, la producción, la estrategia, la planificación o la gestión.

La influencia de las TIC en el sector turístico es de una importancia trascendental hasta los días de hoy. En los momentos actuales las TIC se muestran como un reto y ente activo en el ámbito turístico, contribuyendo con calidad a mantener un desarrollo turístico sostenible gestionando servicios de excelencia. Por tanto, existe la necesidad de valorar el impacto de las tendencias que en materia de TIC el sector del turismo esté a la altura de los nuevos retos y con una visión más humanista en la valoración de los impactos que desde la ciencia y la tecnología se generan, por la elevada significación en el aseguramiento económico de la sociedad que tiene este sector propiciando la sostenibilidad del desarrollo.

\section{Metodologia.}

Enfoque Ciencia, Tecnología y Sociedad 
Debemos establecer que la ciencia y la tecnología son procesos sociales, con un carácter complejo donde median valores culturales, políticos y económicos que se relacionan entre sí para la configuración de un sistema social determinado.

Existe una diferencia marcada entre ciencia y tecnología, que está intrínsecamente ligada con el objetivo de este trabajo. La ciencia persigue conocer desde una visión objetiva la verdad, mientras que la tecnología tiene un fin más bien utilitario y facilista.

La ciencia fomenta la creatividad tecnológica y racionaliza la práctica tecnológica ya existente. Por otra parte, la tecnología contribuye a la ciencia creando instrumentos, poniendo problemas científicos y creando nuevos modelos conceptuales para la ciencia posterior.

Es, no obstante, una reacción que no se agota en los ámbitos social y político. Originarios de finales de los años 60 y principios de los 70, los estudios CTS reflejan en el campo académico y educativo esa nueva percepción de la ciencia y la tecnología y de sus relaciones con la sociedad. (López Cerezo, 1998)

Los años sesenta enmarcaron el contexto para el comienzo de una serie de estudios que buscaban integrar de manera interdisciplinar los conceptos de sociedad, ciencia y tecnología. Este enfoque trae consigo la postura de que la ciencia es producto de la historia, las mediaciones sociales y la cultura: (...) la ciencia es, ante todo, producción, difusión y aplicación de conocimientos y ello la distingue, la califica, en el sistema de la actividad humana.(Núñez Jover, 2003)

Desde este punto de vista, gracias a los aportes teóricos de la ciencia, la tecnología representa un estadio superior a la noción de técnica. Teniendo en cuenta lo anterior, la tecnología está sujeta a un esquema que podemos denominar como determinismo social, lo que nos permite inferir que se moldea en la interdependencia de las estructuras, infraestructuras y superestructuras de los distintos sistemas sociales. Podemos decir que solo la interdisciplinariedad da posibilidad de comprender los fenómenos sociales en su correcto sentido y contexto.

Las consecuencias políticas y sociales del estudio y aplicación de la energía nuclear, las TIC, la carrera armamentística, la medicina, son algunos ejemplos del impacto social de la tecnología en los estilos de vida, en el ámbito interpersonal, en los valores y en las relaciones de poder.

En esta era que vivimos la tecnología es una red que abarca los más diversos sectores de la actividad humana un modo de vivir, de comunicarse, de pensar, un conjunto de condiciones por las cuales el hombre es dominado ampliamente, mucho más que tenerlos a su disposición. (Agazzi, 1996)

Existe una nueva forma de sobrenaturaleza que depende en gran medida de una serie de innovaciones tecnológicas. Está posibilitado por una serie de tecnologías, entre las cuales 
mencionaremos siete: el teléfono, la radio, la televisión, el dinero electrónico, las redes telemáticas, los multimedia y el hipertexto. (González, Cerezo y Luján, 1996)

La construcción y el funcionamiento de cada uno de esos artefactos presupone numerosos conocimientos científicos y tecnológicos (electricidad, electrónica, informática, digitalización, óptica, compresión, criptología, entre otros), motivo por el cual conviene subrayar que esto sólo ha comenzado a ser posible para los seres humanos tras numerosos avances científicos y técnicos. Es por ello que: ... es uno de los resultados de la tecnociencia, y por ello ha emergido en aquellos países que han logrado un mayor avance tecnocientífico: sobre todo en los Estados Unidos de América, en donde se descubrieron, o cuando menos se implementaron y difundieron, la mayoría de esos avances tecnocientíficos. (González et al., 1996)

En la sociedad actual, existe una creencia muy difundida que considera que la tecnología es capaz, prácticamente por sí misma, de incidir de manera directa y positiva en el desarrollo social y económico de un contexto particular. Al respecto el compañero Fidel Castro expresó: Cuba también cuenta con una fuerte tradición de crítica y resistencia al orden mundial actual y al "pensamiento único", especialmente penetrante en la crítica al "capitalismo realmente existente" y especialmente esclarecedor respecto a la marginalidad creciente que las tendencias actuales reservan al tercer mundo. Ese pensamiento se articula coherentemente con el énfasis en el desarrollo de la ciencia y la tecnología y la orientación de estas a la atención de necesidades sociales relevantes. (Castro Ruz, 1990)

La introducción de las TIC en prácticamente cualquier proceso, es sinónimo de productividad y, por ende, de progreso. Esta concepción ha adquirido un valor simbólico tan importante, que suele ser retomada de forma totalmente acrítica por muchos sectores de la sociedad.

Todo esto significa que la tecnología no solo es el resultado de mecanismos y de decisiones estrictamente técnico-científicas, sino que recibe efectos de procesos sociales externos al campo científico tecnológico. Sin embargo, esto no implica que la técnica no cuente con el grado de autonomía necesario y suficiente como para que ciertos cambios $\mathrm{y}$ transformaciones se realicen con independencia de los mecanismos sociales.

En este contexto, el concepto de sociedad de la información, como construcción política e ideológica, se ha desarrollado de la mano de la globalización neoliberal, cuya principal meta ha sido acelerar la instauración de un mercado mundial abierto y autorregulado.

Se considera, por tanto, la visión de la tecnología como motor del progreso, sin embargo, esta visión es un tanto lineal: parte de la noción de progreso científico, el cual conlleva al progreso tecnológico, trayendo consigo lo que consideraría como proceso económico, de ahí el progreso social y finalizaría el ciclo con el progreso científico nuevamente. 
En síntesis, la globalización ha fomentado un sistema económico dominante que tiene en el procesamiento y la comunicación de información su factor estratégico, dejando atrás el viejo esquema económico industrial que giraba en torno al dominio de las fuentes de energía. Hoy, todos los ámbitos de la economía se han visto afectados de manera indirecta o directa por el uso de las TIC.

La centralidad que las tecnologías ocupan en esa concepción de la sociedad resulta desproporcionada y paradójica en países subdesarrollados que no cuentan con los recursos para acceder al llamado progreso tecnológico y que sufren entonces de un ensanchamiento de la brecha digital y por ende la pérdida de confianza en sus sistemas sociales. La contradicción radica entonces en el axioma: sociedad informada vs sociedad informatizada.

El concepto de sociedad de la información en la actualidad tiende a concebirse en términos de la implantación más o menos generalizada de las nuevas tecnologías y fundamentalmente de la popularización de la tecnología informática y el acceso a Internet. Para Carrera (2008) informatización e información empiezan a coincidir casi perfectamente en idéntico molde o forma conceptual.

Es necesario hablar de un paradigma de desarrollo que asigna a la tecnología un rol causal en el ordenamiento social, ubicándola como motor del desarrollo económico. Para los países en desarrollo, este discurso implica que la transición hacia la sociedad de la información es esencialmente una cuestión de tiempo y de decisión política para crear las condiciones adecuadas.

Algo parecido sucedería con relación a los sectores sociales afectados por la brecha digital, a los cuales habría que incluir mediante programas de acceso universal. Al colocar a la tecnología en el centro de este modelo, la industria de telecomunicaciones aparece como la llamada a liderar su desarrollo; mientras que la industria productora de servicios y contenidos digitales asume una influencia inédita. Como se puede apreciar, esta concepción de sociedad, marcadamente neoliberal, refuerza la visión tecnocéntrica de la realidad.

Se habla de un proyecto de convergencia de soportes, lógicas, industriales, culturas organizacionales, mercados y reglamentaciones de las principales industrias relacionadas con la producción, tratamiento, procesamiento, almacenamiento y distribución de información.

Persiste, entonces, la transformación de los procesos productivos, las rutinas profesionales afectadas por la diseminación global de las tecnologías que permiten procesar y comunicar volúmenes de información masivos.

La naturaleza intangible de muchos de los intercambios económicos (básicamente los financieros) que afectan la estructura productiva y la performance de países y regiones 
enteras del planeta, se retroalimenta con las tendencias globalizadoras del capital, (...) línea de aproximación a la economía política de la comunicación. (Rubira, 2009)

La información es un bien público, no una mercancía, la comunicación un proceso participativo e interactivo, el conocimiento una construcción social compartida, no una propiedad privada, y las tecnologías un soporte para todo ello, sin que se conviertan en un fin en sí.

Tendencias tecnológicas en el turismo actual

El desarrollo turístico está conectado cada vez más al crecimiento de las nuevas tecnologías de la Informaión y las Telecomunicaciones, debido al impulso que proporcionan las mismas en los proesos de reservas hoteleras, para la adquisición de determinados productos y servicios.

Desde los años 70 las tecnologías de la información y las comunicaciones (TIC) han transformado el mercado turístico de manera sustancial, pero ha sido desde el año 2000, con los efectos de la llegada de Internet, cuando ha comenzado la verdadera transformación. (Verdecia, 2018)

El objetivo era propiciar la velocidad y los volúmenes de venta a tráves de los nuevos sistemas de reservas. De una forma u otra las TIC resuelven por una parte problemas asociados al desarrollo de la demanda y mejoran el modelo de negocio de cualquier entidad turística, así como que automatizan sus operaciones dando paso a la confección rápida de diversos productos que son ubicados en el mercado para los clientes y que cada vez se convierten en productos competitivos.

Los mercados por su parte crecen en amplitud y en profundidad, surgen mercados emergentes, para lo cual la intermediación busca una solución reduciendo los caminos para la reserva. Las TIC facilitan la operación de cualquier empresa destinada al turismo, pero esto no significa que se obtengan los resultados deseados en tanto no contribuya al incremento de clientes, la rentabilidad del negocio y al aumento de los ingresos. También mediante las TIC se pueden trazar estrategias de acuerdo a objetivos planteados y teniendo en cuenta estadísticas de determinados Sistemas de Gestión Hoteleros.

El producto turismo por su parte engloba una serie de atributos que son gestionados por actores dentor del destino, mediante tecnologías de la distribución que son diferentes para cada uno de sus componentes. El uso de tecnología es crucial para hacer más efectivo y eficiente cualquier negocio; no solo se debe tener en cuenta las necesidades de los clientes sino la de los productores de tecnologías.

El creciente impulso de la Ciencia y la Tecnología, ha logrado tener acercamientos con lugares que jamás se ha imaginado el hombre y se la ve como una técnica de vital importancia para el sector del turismo. (Mullo, Mendoza y Yumisaca, 2018) 
Las TIC son de gran interés para los clientes, productores de servicios y para el destino en general, las tecnologías de las información y las telecomunicacione son importantes para disminuir costes, aumentar la experiencia del cliente, aumentar las ventas y mejorar la eficacia y eficiencia en la gestión y en procesos de trabajo.

La visión de en cuanto a desarrollo de las TIC en el turismo va encaminada buscar dentro del mercado el crecimiento en amplitud, crecimiento en profundidad, búsqueda de valor añadido, aumento de la distancia entre mercados ricos y pobres, disponibilidad, seguridad, garantía, confianza, asistencia en destino, búsqueda del reconocimiento, propagación de la marca, diferenciación en cuanto a precio.

En cuanto a producto se refiere existe una tendencia a un mayor componente de servicio, mayor valor añadido, procesos más complejos, competencia en precios, maduración más rápida, etc. La empresa debe por su parte ir creando condiciones y llevando a cabo acciones que le permitan disminuir márgenes, disminuir ciclos de producción, realización de alianzas estratégicas, aumento de su funcionalidad dentro del mercado y otros.

Hay que tener en cuenta que en la actualidad el segmento de mercado altos ingresos está en aumento y se deben crear las condiciones para ello, donde juega un papel fundamental el crecimiento y fomento de nuevas tecnologías de la Información y las comunicaciones que faciliten los procesos en cada negocio dedicado a la actividad turística.

El uso de las TIC en el turismo garantiza la adecuada prestación de los servicios al cliente final por ejemplo la utilización de oficinas de turismo virtuales, pagos por móviles, checkin vía móvil, consumidores multipantalla, gestión integral (Paperless), marketing digital, entre otros.

El mercado turístico no solamente se ha visto fuertemente afectado por la brusca incorporación de Internet, sino también por otras tecnologías emergentes (realidad virtual, realidad aumentada, big data, conectividad, internet de las cosas, robótica, entre otras) que han modificado los canales de comercialización y distribución, han creado nuevos modelos de negocio, han cambiado la forma de ofrecer experiencias y personalizar la oferta, lo cual resulta esencial a la hora de entender el nuevo entorno. (Albornoz, 2020)

Todo lo antes mencionado forma parte de un conjunto de mecanismo que las tecnologías proporcionan para optimizar el funcionamiento de cualquier empresa destinada a la distribución de productos-servicios.

El desarrollo de las TIC ha sido el germen de importantes cambios tanto desde el lado de la oferta como de la demanda, con grandes efectos sobre la situación competitiva de las empresas de servicios, en general, y de las empresas del sector turístico en particular. La innovación y la utilización estratégica de la tecnología en el sector turístico alcanzan su mayor exponente en las centrales de reservas de las compañías aéreas (CRS). 
La expansión de las actividades y la inclusión de otros muchos productos turísticos y formas alternativas de distribución, con un acceso on line, han derivado desde los CRS iniciales hacia los actuales Sistemas Globales de Distribución (GDS) Ej. Galileo, Sabre, Amadeus, Worldspan. A las funciones que antes proporcionaban los CRS se unen nuevas prestaciones como: base de datos de clientes, agendas, conexión con los programas de gestión interna y ventas o la emisión del billete electrónico. Este nuevo concepto de sistema de reservas supuso una globalización tanto en los servicios ofrecidos como en los mercados geográficos en los que se operaba.

Dentro de todo el proceso viene a jugar un papel importante el uso del Internet pieza tecnológica que va incluida dentro de la arquitectura de venta permitiéndole al cliente pagar mediante vía online determinado producto o servicio. El Internet permite tanto a los productores de servicios como al cliente un camino más rápido para el conocimiento de determinado producto, así como la compra del mismo, lo cual no quita que existan compradores que desconfíen de esta opción y que prefieran todavía la venta directa.

El turismo se puede desarrollar de muchas formas, una de ellas es visitar los lugares de interés para el turista, pero ahora gracias al apoyo que nos brinda la tecnología se pueden conocer estos lugares así nos encontremos al otro lado del planeta, un claro ejemplo de esto son las nuevas e innovadoras visitas virtuales que las personas pueden llevar a cabo a través del Internet, valiosa y magnifica herramienta que nos permite realizar infinidad de cosas como vender, comprar, conocer, hablar con familiares y muchas otras funciones.

La aparición de Internet como nuevo canal de distribución, ha transformado los procesos y funciones de intermediación que venían desarrollando distintos actores tales como los operadores mayoristas. Aunque la posibilidad de la relación directa entre los proveedores y prestadores de los servicios y el cliente final siempre ha existido, la consolidación de Internet es determinante. Tal es así que podríamos afirmar que la incorporación de las nuevas tecnologías de información y comunicación ha modificado la naturaleza misma de la cadena de valor de los servicios turísticos. Este nuevo escenario reclama la resignificación de los agentes que participan de todos los circuitos y procesos de intermediación, ya sea en la comercialización como en la promoción y la reserva.

Más de 50 años han transcurrido hasta hoy en día, en los que el comercio electrónico ha evolucionado desde la antigua venta por catálogo de los años 80, a la aparición de la world wide web (www) en los años 90, la revolución de las puntocom en el año 2000 o el desarrollo de las comunicaciones $3 \mathrm{G}$ y $4 \mathrm{G}$ que han conllevado la expansión masiva del uso del Smartphone. (Macías, 2020)

Uno de los modelos utilizados dentro de las nuevas Tecnologías de la Información y las Comunicaciones son los programas de afiliación y el marketing viral. La importancia de estos programas radica en que cualquier entidad que quiera aplicarlo permite ofrecer al cliente información sobre vuelos, hoteles, actividades, restaurantes, conciertos, guías, fotos, videos. Además, integrar experiencias de viaje de amigos y amigos de amigos en redes sociales, encontrar los mejores precios. La visión que se tiene en cuanto al tema es 
el gran crecimiento de programas de afiliación entre aerolíneas y proveedores de alojamiento en los últimos años.

Muchas son las investigaciones actuales sobre las nuevas tendencias turísticas. Bonilla, (2013) ha investigado sobre los nuevos estilos turísticos. A mediados del S.XX apareció un nuevo concepto turístico llamado turismo "New Age" basado en el viaje turístico como aprendizaje, conectando con la gente local del destino.

El estudio contribuye a la literatura a visualizar la forma de operar con los sitios Web, el contenido y su estructura, la capacidad de cambio de los contenidos, así como la velocidad de cambio de la información en línea, entre otros. Todos estos elementos influyen significativamente en el valor percibido por los consumidores, y son articuladores en relación a los efectos determinantes en la intención de continuar con el uso de servicios de turismo en línea. (Bonilla, 2013)

Por las particularidades del sector turístico, éste se constituye como adecuado para la medición de sus acciones de marketing online o presencia en medios sociales, ya que la subjetividad que recorre todo el proceso turístico anima a incluir herramientas de medición que tengan en cuenta este hecho. (Bonilla, 2013)

En Cuba, Internet y las redes sociales han transformado las formas de viajar y disfrutar los viajes, el fortalecimiento de las infraestructuras de conectividad de las instalaciones turísticas para la gestión en estos medios; así como el despliegue de cobertura WiFi, 3G y 4G, constituyen también evidencias de la transformación digital del destino. Aspiramos llegar a la fase superior de la transformación digital, "Los Destinos Turísticos Inteligentes", donde la Accesibilidad, Sostenibilidad, Innovación, Gobernanza y Tecnologías se integran para una gestión eficiente de los recursos turísticos del país" (Redacción Adelante, 2018)

La Sociedad Estatal para la Gestión de la Innovación y las Tecnologías Turísticas de la Secretaría de Estado de Turismo (SEGITTUR), define los destinos turísticos inteligentes como destinos turísticos innovadores, consolidados sobre una infraestructura tecnológica de vanguardia, que garantizan el desarrollo sostenible del territorio turístico, accesible para todos, que facilitan la interacción e integración del visitante con el entorno e incrementan la calidad de su experiencia en el destino. (Gil, Fernández y Herrero, 2015)

En el Taller sobre las TIC al servicio del Comercio realizado durante la II Convención de Comercio Cuba 2019, el Dr. José Enrique Salgado, Profesor Titular de la Facultad de Turismo, Universidad de La Habana y Gerente de la Sucursal Cuba del Grupo Amadeus I, analizó el uso que actualmente hacen los viajeros de las nuevas tecnologías durante las visitas tanto culturales, de sol y playa, o negocios.

De acuerdo con los datos citados por el experto, ocho de cada diez personas, (el $81 \%$ ) utiliza Internet para informarse sobre los lugares a visitar, muchos de ellos reservan y compran online; uno de cada cuatro internautas utiliza aplicaciones móviles de 
geolocalización; el 64\% usa los medios sociales para compartir experiencias, fotos o videos y la mayoría lo hace desde un smartphone. (Redacción TTC, 2019)

Entre otras ventajas que se pueden ofrecer al visitante de la nueva era, las tecnologías que se demanda en un Destino Turístico Inteligente se encuentran WiFi gratuito, apps para smartphones, la posibilidad de realizar pagos vía móvil, el uso de códigos QR, de realidad aumentada en los sitios de interés, monedas virtuales, webs dedicadas a ese tipo de destino. Entre los destinos turísticos cubanos, Cayo Largo y Cayo Santa María dan los primeros pasos en este sentido para gradualmente extenderlo al resto de los destinos de la Isla.

En la nueva era del turismo digital, la inteligencia turística entra de lleno en los destinos y organizaciones que quieran incorporar la gestión de la información en el día a día, para una toma de decisiones estratégica, gestión del Big Data y Open Data. La implantación de Sistemas de Inteligencia Turística permite potenciar la competitividad del destino, organizaciones y conocer mejor al visitante.

Se define como sistema de inteligencia turística a aquella herramienta tecnológica que permite cargar, procesar y analizar datos de diferentes fuentes de información, tanto propias como externas, de manera que estos datos se transformen en conocimiento relevante y que ayude en el proceso de gestión de un destino o una empresa. (Albornoz, 2020)

La Empresa para el Desarrollo de Aplicaciones de Tecnologías y Sistemas (DATYS), propuso en FitCuba 2019, un proyecto integrador que aglutina a las entidades del país (fundamentalmente empresas del sector turístico como agencias de viajes, hoteles y el MINTUR) para que aporten sus ideas con el objetivo de conseguir un salto de calidad del producto turístico cubano. Este es un proyecto colaborativo que contribuye a la construcción de un destino seguro sin grandes inversiones. Según refleja Gómez, (2019) este proyecto trata de hacer análisis de la información, la cual hoy está segmentada y cada cual la analiza a su manera. DATYS puede brindar herramientas y obtener análisis inteligentes de esa información y ponerla de manera inmediata en conocimiento de las autoridades para aplicarlas, tomar decisiones a corto, mediano y largo plazo.

La influencia de las Tecnologías de la Información y las Comunicaciones (TIC) en el sector turístico y en especial en la comunicación de los productos y en la gestión de las relaciones con los públicos es ampliamente conocida. Las aplicaciones Web 2.0 basadas en comentarios y valoraciones de usuarios a escala mundial, en concreto TripAdvisor, influencian las prácticas en relaciones públicas y comunicación turísticas obligando a una cuidada gestión de la reputación online. Si por un lado las nuevas herramientas 2.0 están a disposición de las organizaciones para comunicar con sus públicos, por otro lado, son también fuente de comentarios y valoraciones directas que pueden reforzar positivamente. (Verdecia, 2018) 
Internet ha mejorado el sector empresarial de forma radical, tal es el caso que se han eliminado las barreras geográficas, se puede vender los 7 días de la semana y las 24 horas del día, los consumidores tienen una amplia oferta de productos y servicios que comparar y elegir, además, los medios de pago se han adaptado a la evolución de Internet y se puede realizar cualquier compra tranquilamente desde casa. El sector del Turismo ha sabido aprovechar al máximo estas ventajas y se ha posicionado como el número uno dentro de las ventas online desde hace ya años.

\section{Resultados.}

El mercado ha sufrido cambios y actualmente marcan las nuevas tendencias del turismo con el aprovechamiento de las tecnologías de información y de las comunicaciones. El turismo ha evolucionado desde sus inicios hasta la actualidad. Según expresa Bonilla, (2013): hay principalmente tres factores que han generado cambios en el sistema turístico. Primero, un cambio en la visión social del mundo y del comportamiento del ser humano en su entorno, factor que está más que todo relacionado con lo sociológico y lo natural. Segundo, la evolución de los medios de transporte y gracias también a las nuevas tecnologías. Tercero, el surgimiento de nuevos medios de comunicación y de la tecnología como motor de cambio.

Las TIC en el turismo les permiten a las empresas de hoy en día:

1. Tener una presencia global en el mercado internacional.

Las TICS permiten el acceso a las principales plazas turísticas, además posibilitan el acceso a otros productos paralelos, como los mapas de carreteras, la información del tráfico, los consejos al viajero, la meteorología y otros. La presencia en Internet no es solamente un negocio on line, sino permite la inclusión en el mercado de otros factores como la imagen, la publicidad o el ofrecer servicios de valor añadido a sus clientes habituales.

2. Investigar y desarrollar nuevos productos turísticos.

Estos productos deben satisfacer la demanda de determinados nichos de mercado, alcanzando una ventaja competitiva a través de la especialización y de la diferenciación.

Las TIC a través de la flexibilidad permiten variadas combinaciones a los consumidores para diseñarse su propio producto turístico. Así, las empresas van a poder individualizar su producto para cada uno de sus clientes, permitiéndoles que seleccionen las características que prefieran para dicho producto.

3. Flexibilizar precios.

Estos precios deben ser más competitivos y optimizando su producción.

A través de las TIC se puede supervisar las ventas en tiempo real, las empresas turísticas pueden actuar llevando a cabo correcciones en la configuración del producto o en su precio, o incluso acudir a la publicidad para maximizar sus ventas. Las TIC alertan a las empresas turísticas sobre posibles excesos en la demanda o en la oferta, y dado que el producto turístico por su propia naturaleza no es almacenable para un 
consumo posterior en el tiempo, permiten realizar ofertas de última hora que eviten que cierta cantidad de este producto quede sin vender.

4. Ahorrar tiempo.

Las TIC permiten a las empresas turísticas compartir información internamente o con sus asociados de forma instantánea, aumentando su eficiencia y a los consumidores interactuar recíprocamente con estas empresas, realizando confirmaciones y compras de forma inmediata.

Al poder realizar transacciones con los proveedores de servicios turísticos las 24 horas de todos los días del año, sin necesidad de su presencia física, lo que supone una ventaja competitiva para éstas.

Las TIC eliminan las barreras geográficas, por lo que permiten aumentar la base de clientes potenciales que de otra forma no podrían contratar los servicios de estas empresas debido al factor distancia. Las nuevas tecnologías van a facilitar la oferta conjunta de productos turísticos con otros que no lo son. A través de portales la empresa turística puede servir de vía de acceso de otros productos de comercio electrónico que de otra forma verían reducidas sus posibilidades de éxito comercial.

Las TIC reportan numerosas ventajas al consumidor de productos turísticos, entre las que están la mayor transparencia de precios, la posibilidad de una oferta personalizada, una mejor información visual y gráfica (videos, fotos, mapas, planos, productos interactivos...), inexistencia de barreras geográficas y temporales, mayor poder de decisión y formación, facilidad para comparar entre diferentes productos, ahorro de tiempo, menor necesidad de anticipar las reservas y productos más baratos gracias al recorte de costes de producción.

Las empresas turísticas deberán desarrollar estrategias multicanal, que incluyan a todos los intermediarios del nuevo turismo electrónico (Internet, telefonía fija y móvil, y televisión digital) para poder responder adecuadamente a las exigencias del mercado.

Por todo lo anterior es evidente que las TIC modificarán el futuro de la intermediación turística, lo que se aprecia en diversas tendencias actuales como son el hecho de que los intermediarios tradicionales están reestructurando sus procedimientos para actualizar su oferta, mejorar la satisfacción de sus clientes y mantener la competitividad; los destinos turísticos desarrollen sistemas regionales para mejorar su representatividad, potenciar su imagen y atraer reservas directas; o que los proveedores de productos y servicios turísticos estén creando sistemas basados en Internet para comunicarse directamente con sus clientes.

Las nuevas tecnologías de la información y de la comunicación van a provocar importantes ajustes en los que se tenderá acortar el canal de distribución, nacerán nuevos tipos de intermediarios y desaparecerán los que no sean capaces de adaptarse a la nueva situación generada por éstas. 
A lo largo de los años la tecnología ha ido irrumpiendo en nuestras vidas y en todos los sectores. Los turistas van a agradecer que este elemento esté presente durante el viaje sobre todo para darle más practicidad a las cosas y hacerlas más sencillas.

La posibilidad de usar tu teléfono para acceder sin llaves a la habitación de tu hotel, recibir consejos de viaje personalizados o hablar en tu idioma con un robot recepcionista serán posiblemente ya realidad este año. Las innovaciones impulsarán la sencillez para mejorar la seguridad y flexibilidad de los viajes. (Reñones, 2020)

En la actualidad los consumidores actúan por sí solos en la búsqueda de información sobre hoteles, vuelos, paquetes turísticos, opciones recreativas y gastronómicas, abriendo un camino bidireccional, en el cual la decisión final está matizada por ese intercambio inicial en las páginas webs.

Las TIC han ayudado al turismo en:

- Facilitar la distribución de información y productos turísticos por Internet.

- Mejorar toda comunicación basada en la promoción, publicidad, comercialización y contratación de servicios turísticos.

- Ha creado la necesidad de contar con mecanismos de difusión, promoción, comercialización y desarrollo para todo tipo de cliente, a través de medios de comunicación.

- Ofrecer productos y servicios turísticos directamente a un gran número de consumidores a un costo relativamente bajo y mantener relaciones con ellos, así como con otros productores y distribuidores.

- El turismo busca atraer clientes internacionales, lo que exige un desarrollo importante en materia de infraestructura, en comunicaciones que permitan y faciliten la promoción y comercialización de los atractivos turísticos en todo momento y en cualquier lugar del planeta.

- El turismo depende extraordinariamente de las TIC, pues antes de realizar un viaje, los turistas demandan información para planear y elegir entre múltiples opciones, y también observar la necesidad creciente de información durante y después del viaje.

- Las organizaciones han adquirido las TIC para implementar y actualizar continuamente tecnologías en sus instalaciones con el fin de mantener una ventaja competitiva mediante la innovación de sus productos y de esta manera poder competir en el mercado.

También ha cambiado la forma en que los consumidores planifican y compran sus vacaciones, ahora el turista puede conocer de información sobre vuelos, hoteles, restaurantes, paquetes turísticos y métodos de pago, desde la comodidad de su casa o trabajo y sin tener que salir para hacer todo eso. (Mullo, Mendoza y Yumisaca, 2018) 
Con la facilidad que tiene cualquier persona de acceder a la informacion hoy en dia, el mercado turístico cuenta con diversas herramientas para promocionar sus productos, cosa que era un poco dificil en años anteriores ya que la tecnologia estaba limitada. Las Tecnologías de Información y Comunicaciones han establecido un mayor grado de colaboración con empresas e instituciones, yendo un paso más allá del perfil tradicional de la empresa de servicios. Los principales beneficios de las TIC se obtienen cuando ellas se unen con otros activos organizativos y en este sentido el resultado de este proceso no solo depende de la intensidad en el uso de las nuevas tecnologías sino también en la manera en que se organice este uso y en la calidad del entorno en el que opera la empresa.

Algunos de los impactos que han tenido las TIC en el turismo cubano podemos decir que:

- Hay presencia en Internet y en las redes sociales de la gran mayoría de las empresas del sector de alojamiento y de las agencias de viajes ya que tienen su propia web y en algunas se permite las reservas online.

- Se usan para promocionarse y como marketing para desarrollar su imagen corporativa.

- Los operadores de transporte también han hecho uso de las TIC en el sector, con hitos relevantes como la venta y facturación del pasaje electrónico mediante sus propias webs.

- La tecnología móvil y los servicios basados en geolocalización se extienden ya que también se puede hacer uso de servicios de información sobre la situación de los vuelos, entre otros.

- En el sector alojamiento y alquiler de vehículos se extienden las tecnologías que posibilitan el registro de entrada y salida o la recogida del auto mediante la web o el móvil.

- Permite acceder y compartir contenidos en tiempo real desde cualquier lugar en el que nos encontremos gracias al uso del móvil durante el viaje, ya sea en la planificación o a lo largo de éste.

\section{Conclusiones.}

- El creciente impulso de la Ciencia y la Tecnología, ha logrado una evolución y avance de la tecnología en el sector turismo, el cual se ha visto bastante beneficiado ya que en años anteriores el flujo de información era muy escaso.

- Dentro del ámbito del turismo, el uso de las TIC ha crecido en los últimos años, convirtiéndose en una herramienta fundamental para atraer a turistas por parte de los diferentes destinos y empresas turísticas.

- El internet ha cambiado los hábitos de los usuarios en la búsqueda, planificación y compra de sus vacaciones y está transformando los modelos de negocio y la forma de promocionar y vender los productos y los servicios, que los diferentes proveedores turísticos desarrollan en el mercado. 


\section{Referencias bibliográficas.}

Agazzi, E (1996). El bien, el mal y la ciencia. Madrid, Editorial Tecnos, S.A.

Albornoz, Á. C. de (2020). EBOOK "SISTEMA DE INTELIGENCIA TURÍSTICA". España. La Plataforma Tecnológica del Turismo, THINKTUR.

Bonilla, J (2013). Nuevas tendencias del turismo y las tecnologías de información y las comunicaciones. Universidad Externado de Colombia.

Carrera, P (2008). Teoría de la comunicación mediática. Valencia. Editorial Tirant Lo Blanch.

Castro Ruz, F (1990). Ciencia tecnología y sociedad. La Habana. Editorial Política.

Fernández Álvarez, B (2015). TIC y Turismo. Recuperado 17 de julio de 2020 de http://www.hosteltur.com/como-seran-destinos-turisticos-exito-futuro.html

Gil, AML., Fernández, BZ. y Herrero, JLC (2015). Los Destinos Turísticos Inteligentes en el marco de la Inteligencia Territorial: conflictos y oportunidades. Revista Investigaciones Turísticas.

Gómez,C (14 de Mayo de 2019). DATYS: Un destino inteligente es siempre seguro. Excelencias Cuba. https://www.excelenciascuba.com/fitcuba/datys-un-destinointeligente-es-siempre-seguro

González García, MI., Cerezo, JAL. y Luján, JL (1996). Ciencia, Tecnología y Sociedad: una introducción al estudio social de la ciencia y la tecnología. Madrid. Tecnos.

López Cerezo, JA (1998). Ciencia, tecnología y sociedad: el estado de la cuestión en Europa y Estados Unidos. Revista Iberoamericana de Educación. 18.

Macías, A (2020). El Comercio Efectivo. Recuperado 17 de julio de 2020 de https://www.andalucialab.org/ blog/como-internet-ha-revolucionado-el-sectordel-turismo/

Mullo Romero, E., Mendoza Tarabó, E. y Yumisaca Tuquinga, J (2018). El impacto de la ciencia y tecnología en la cultura del turismo comunitario. Revista Ciencias Pedagógicas e Innovación.

Núñez Jover, J (2003). La Ciencia y la Tecnología como procesos sociales. Lo que la educación científica no debería olvidar. La Habana. Editorial Félix Varela.

Redacción Adelante. (27 de Septiembre 2018). Cuba trabaja por destinos turísticos inteligentes. Adelante. http://www.adelante.cu

Redacción TTC. (16 de Mayo de 2019). Destinos y ciudades inteligentes: el turismo del 
futuro. TTC. https://www.traveltradecaribbean.es/destinos-y-ciudadesinteligentes-el-turismo-del-futuro

Reñones, S (2020). ¿Cuáles son las tendencias turísticas para el 2020?. Recuperado 17 de julio de 2020 de https://www.ceupe.com

Rubira, R (2009). Comunicación, Tecnología y Sociedad. Selección de lecturas. La Habana. Editorial Félix Varela.

Verdecia Rosales, A (2018). Tendencias del consumidor digital para el producto turístico. Revista Granmense de Desarrollo Local. Vol.2.

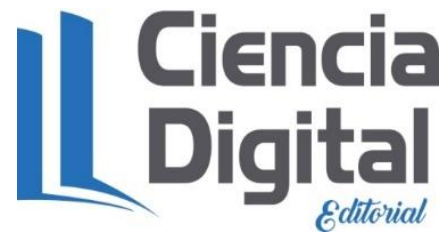




\section{Para citar el artículo indexado}

Nuñez Torres, E., Pérez Rives, L., Montes de Oca Companioni, E. T., \& Velastegui López, L. E. (2021). Estudios CTS sobre las tendencias de las Tecnologías de la Información y las Comunicaciones aplicadas al Turismo . AlfaPublicaciones, 3(2.1), 138-155. https://doi.org/10.33262/ap.v3i2.1.54

\section{Ciencia Ligital}

El artículo que se publica es de exclusiva responsabilidad de los autores y no necesariamente reflejan el pensamiento de la Revista Alpha Publicaciones.

El artículo queda en propiedad de la revista y, por tanto, su publicación parcial y/o total en otro medio tiene que ser autorizado por el director de la Revista Alpha Publicaciones.
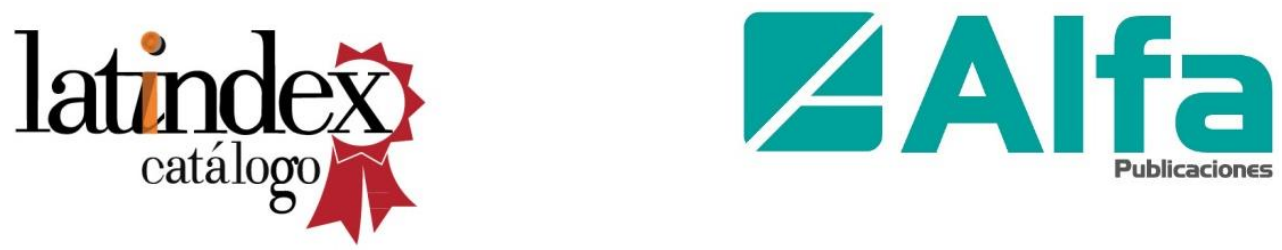